\title{
Is spin-charge separation observable in a transport experiment?
}

\author{
T. Ulbricht ${ }^{1}$ and P. Schmitteckert ${ }^{2}$ \\ 1 Institut für Theorie der Kondensierten Materie - Universität Karlsruhe, 76128 Karlsruhe, Germany \\ ${ }^{2}$ Institut für Nanotechnologie - Forschungszentrum Karlsruhe, 76021 Karlsruhe, Germany
}

\begin{abstract}
We consider a one-dimensional chain consisting of an interacting area coupled to non-interacting leads. Within the area, interaction is mediated by a local on-site repulsion. Using real time evolution within the Density Matrix Renormalisation Group (DMRG) scheme, we study the dynamics of wave packets in this two-terminal transport setup. In contrast to previous works, where excitations were created by adding potentials to the Hamiltonian, we explicitly create left moving single particle excitations in the right lead as the starting condition. Our simulations show that such a transport setup allows for a clear detection of spin-charge separation using time-resolved spin-polarised density measurements.
\end{abstract}

PACS numbers: 71.10.Pm,71.10.Fd,72.15.Nj

\section{Introduction}

Although spin-charge separation (SCS) is undisputed to happen in one-dimensional systems, its direct experimental observation and verification is a subject of many works over the past years. In one dimension, the Fermi liquid picture of non-interacting quasi-fermions is not appropriate [1]. Instead, in the paradigmatic TomonagaLuttinger model and in the prototypical Hubbard model collective bosonic spin and charge excitations are the lowenergy excitations. These so-called spinons carry spin but no charge and so-called holons carry the charge but no spin and spinons and holons may propagate with independent velocities.

Up to now few experimental setups barely show direct evidence of separate spin and charge excitations [2]. Beside tunnelling experiments e.g. momentum-conserved tunnelling on cleaved-edge overgrown GaAs/AlGaAs heterostructures [3] and single-point tunnelling into carbon nanotubes [4, 5], there is angle-resolved photo emission spectroscopy (ARPES) on quasi-one-dimensional $\mathrm{SrCuO}_{2}$ material [ $[6]$ and on the organic conductor TTFTCNQ [7, 8] which seem to show distinct spinon and holon branches in the spectral function. Numerical simulations explaining the latter experiments were done by Benthien et. al. 99].

Here, we propose the following transport experiment: A one-dimensional interacting region is connected to noninteracting leads on both sides. In one of the leads a single electron with average momentum $k_{0}$ is injected into the system. The excitation, by passing through the interacting region, ends up in the other non-interacting lead, where a spin-resolved and time-resolved measurement of charge density is carried out. This setup poses the following questions which we answer in this paper: If one injects an electron with definite momentum at some time $t_{0}$ into an interacting system, where the separation of spin and charge is known to happen, what happens to the electronic excitation within the interacting region? And what kind of excitation emerges from the interacting area at a later time? Will we see distinct excitations in spin and charge density, will we find spin and charge density to be recombined to one full electronic excitation, or will we obtain an incoherent superposition of many excitations?

Safi and Schulz [10] analyse such a transport setup analytically for a spinless Tomonaga-Luttinger liquid model. For the spinful case, however, they have to neglect "umklapp processes and the backscattering of electrons with opposite spin" to sketch a qualitative picture, thus looking at an idealised system, where spin and charge currents are conserved separately. The first numerical simulations for spin-charge separation were performed by Jagla et. al. 11 using exact diagonalisation for 16 sites. Zacher et. al. 12. used Quantum Monte Carlo simulations to study the spin and charge susceptibilities of the Hubbard model. Kollath et. al. [13] presented the first spin-charge separation calculations in the framework of adaptive time dependent DMRG (td-DMRG). This calculation was redone by Schmitteckert [14], keeping up to 5000 states per DMRG block showing that the adaptive time evolution scheme is prone to large numerical errors in the long time limit. In the same line, Schmitteckert and Schneider [15] used over 10000 states per DMRG block to show SCS in a 33 site system with periodic boundary conditions which avoids Friedel oscillations.

In order to avoid the numerical problem associated with the adaptive time evolution scheme and to avoid the numerical costs of a pure full td-DMRG we apply a hybrid of both methods, see [14, 16]. We first use the standard ground state DMRG [17 to obtain our initial state. We then perform typically ten time steps using the full td-DMRG [18] and continue with an adaptive td-DMRG 19, 20, 21] scheme. For recent reviews on DMRG, see [22]. In addition, two main objectives distinguish our work from others that use real-time analysis and model strongly interacting fermionic systems. 1) Previous works either treated only homogeneous interacting systems [11, 13, 18] or modelled optical traps 13. In contrast to that our system is a two-terminal setup modelling a transport experiment. 2) While in earlier works [13, 18] excitations were studied based on the modification of local potentials, we create an excited state by explicitly adding one electron to the system and 
we monitor the time evolution of such an excited state.

Outline First we motivate our creation of an excitation and outline how the numerical method is used to measure the observables. This is accompanied by bulk simulations on a non-interacting and an interacting system. Then we apply the method to the transport setup.

\section{Motivation and method}

An excitation, created at a given site $x_{0}$ in the tightbinding model by using $c_{x_{0}}^{\dagger}$, excites all eigenmodes in the diagonal basis of momentum eigenstates of the tightbinding model. This is not particularly useful since we want to consider electrons with a definite momentum. On the other hand, creating an excitation using $c_{k_{0}}^{\dagger}$ local in the momentum space is again an eigenstate of the system and invariant under time evolution. Therefore in the ground state $|\Psi\rangle$ of a half-filled tight-binding model (for each spin specie $\sigma= \pm \frac{1}{2}$ ),

$$
H_{\mathrm{tb}, \sigma}=-\sum_{x}\left(c_{x-1, \sigma}^{\dagger} c_{x, \sigma}+\text { h.c. }\right)
$$

we create an excitation with spin $\sigma$ by using a Gaussian distribution of creation (annihilation) operators, with momenta centred around $k_{0}>k_{F}\left(k_{0}<k_{F}\right)$ :

$$
\begin{aligned}
g_{\sigma}^{(\dagger)}\left(k_{0}\right) & =\sum_{k} \mathrm{e}^{-\frac{\left(k-k_{0}\right)^{2}}{2 \sigma_{0}^{2}}} c_{k, \sigma}^{(\dagger)} \\
& =\sum_{x=1}^{M}\left(\mathrm{e}^{\left(-2 \sigma_{0}^{2} \pi^{2} x^{2}\right)}\right)\left(\mathrm{e}^{\frac{2 \pi i x}{M} k_{0}}\right) c_{x, \sigma}^{(\dagger)}
\end{aligned}
$$

where $k$ are the momentum eigenstates $k \in\{-\pi+$ $\left.\frac{2 \pi}{M}, \ldots, \pi\right\}$, and $\sigma_{0}$ is the width of the excitation in momentum space. Additionally, the excitation will be centred around $x_{0}$ and normalised such that integrating over all $k$ will resolve to exactly one added electron (hole). A visualisation of this construction is shown in figure 1. An excitation created in this way

$$
\left|\Psi^{+1}\right\rangle=\frac{1}{\sqrt{C}} g_{\sigma}^{(\dagger)}\left(k_{0}\right)|\Psi\rangle
$$

with $C=\left\langle\Psi\left|\left(g_{\sigma}^{(\dagger)}\left(k_{0}\right)\right)^{\dagger} g_{\sigma}^{(\dagger)}\left(k_{0}\right)\right| \Psi\right\rangle$ will move through the system in one direction with a group velocity $v\left(k_{0}\right)=$ $\left.\frac{\partial \epsilon(k)}{\partial k}\right|_{k_{0}}$. Using the Schrödinger picture and evolving our initial state $\left|\Psi^{+1}(t)\right\rangle=\mathrm{e}^{i H t}\left|\Psi^{+1}\right\rangle$, the measured observable is the spin-resolved density

$$
n_{\sigma}(x, t)=\left\langle\Psi^{+1}(t)\left|c_{x, \sigma}^{\dagger} c_{x, \sigma}\right| \Psi^{+1}(t)\right\rangle
$$

which allows the measurement of charge density $n_{c}=$ $n_{\uparrow}+n_{\downarrow}$ and spin density $n_{s}=\frac{1}{2}\left(n_{\uparrow}-n_{\downarrow}\right)$ at any time $t$ as a function of $x$.

In the following figures, we use DMRG [17] to generate the ground state $|\Psi\rangle$ and the excited state $\left|\Psi^{+1}\right\rangle$. For

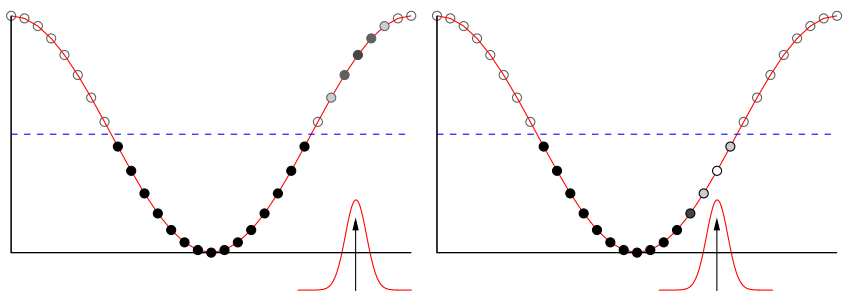

FIG. 1: (Colour online) Visualisation of the creation of an electron (left) or a hole (right) by indicating the occupation of single particle levels in the dispersion relation of the noninteracting problem. The dotted line represents the Fermi energy.

both, the full and the adaptive td-DMRG, the time evolution is performed by a Krylov subspace based evaluation of the time evolution $\left|\Psi^{+1}(t+\Delta t)\right\rangle=\mathrm{e}^{i H \Delta t}\left|\Psi^{+1}(t)\right\rangle$, for details see [14, 16, 18]. The time steps were $\Delta t=0.5$ (in units of time) for both methods.

We use hard-wall boundary conditions in all simulations and all energies are in units of the hopping parameter which is set to 1 in (1). We also calculate the static ground state density $n_{0, \sigma}(x)=\left\langle\Psi\left|c_{x, \sigma}^{\dagger} c_{x, \sigma}\right| \Psi\right\rangle$ at time $t=0$ and form the corresponding charge and spin densities $n_{0, c}, n_{0, s}$. Then we plot the quantities $n_{s}(x, t)-n_{0, s}(x)$ and $n_{c}(x, t)-n_{0, c}(x)$. This trick evens out all stationary oscillations already present in the ground state, like the Friedel oscillations resulting from using hard-wall boundary conditions. Additional $2 k_{F^{-}}$ oscillations resulting from the density distortion of the added excitation are evened out using a three-point average.

In our non-interacting (tight-binding) reference system (11) in figure 2 we create an electron at the real space position $x_{0}=15$ (upper diagram) which travels to the right (lower diagram). The system of size $M=102$ is at halffilling plus one electronic excitation $N_{\uparrow}=51+1$. The $N_{\downarrow}=51$ down-spins are, of course, irrelevant in the noninteracting case and constant in the time evolution. The excitation's momentum is centred around $k_{0}=\pi / 2+0.1$ with width $\sigma_{0}=0.03$. With this width, we ensure that the momentum distribution is far away (compared to the width) from the Fermi surface $k_{0} \sim k_{F}+3 \sigma_{0}$ but still as close as possible to the linear regime of the cosine band at $k_{F}$. The snapshots at $t=0$ and $t=36$ in figure 2 of spin and charge density serve as a proof-of-concept for the numerics. They show the expected synchronous motion of the electronic excitation with group velocity $v_{G}=1.93 \pm 0.02$. A delta-function-shaped excitation in the thermodynamic limit of this system would have a group velocity of $v_{G}\left(k_{0}\right) \sim 1.99$. Since we are in a finite system, the discretisation and the cut-offs at the Fermi energy and the band edge directly influence the actual shape of the initial density distribution. Also, the nonlinearity of the band affects the packet propagation via the width and position of the momentum distribution. The (otherwise perfect time invariant) Gaussian shape is 


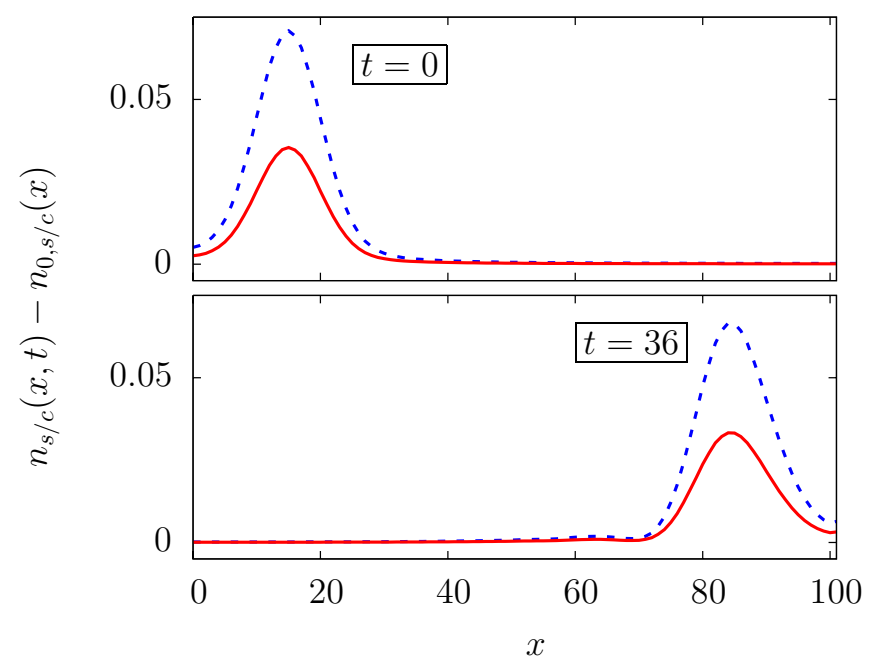

FIG. 2: (Colour online) Spin (solid,red) and charge (dashed, blue) densities of a td-DMRG simulation of one additional up-electron over the background of a half-filled tight-binding model for each spin specie. The Gaussian excitation on the left at time $t=0$ (upper diagram) moving to the right at $t=$ 36 (lower diagram) retains its shape. Averaging is described in the text.

distorted and the packet is slowed down. This system was calculated using $N_{\text {cut }}=1000$ states per DMRG block, applying 10 time steps in the full td-DMRG and keeping 1000 states for the consecutive adaptive td-DMRG. The discarded entropy never exceeds the order of $10^{-5}$. Comparing to an exact diagonalisation (ED) we define the peak relative error in the densities by

$$
\left|\delta n_{p}(t)\right| \equiv \max _{x}\left(\frac{n_{\mathrm{ED}}(x, t)-n_{\mathrm{DMRG}}(x, t)}{n_{\mathrm{DMRG}}(x, t)}\right)
$$

This quantity reaches from initial $\left|\delta n_{p}(0)\right|=7 \cdot 10^{-5}$ peak relative error to $\left|\delta n_{p}(36)\right|=7 \cdot 10^{-3}$ peak relative error at $t=36$.

Now we repeat the simulation in a Hubbard model bulk system

$$
H_{\mathrm{bulk}}=\sum_{\Sigma=\uparrow, \downarrow} H_{\mathrm{tb}, \sigma}+U \sum_{x}\left(n_{x, \uparrow}-\frac{1}{2}\right)\left(n_{x, \downarrow}-\frac{1}{2}\right) .
$$

While the previous excitation was a superposition of well defined eigenstates in the tight-binding model, it is not clear which eigenmodes are excited by adding/removing an electron from an interacting system. Nevertheless, using the same $c_{\sigma x}^{(\dagger)}$ in equation 3 to create an excitation still results in a Gaussian shape in spin and charge densities (see figure 3), although the corresponding Fourier transformed operators $c_{\sigma k}^{(\dagger)}$ no longer create single particle eigenstates of the system. This time we insert an excitation corresponding to one (up-spin) hole into the ground state of a repulsive particle-hole symmetric Hubbard model (6) with $U=4$ at a filling of $\frac{41}{90} \approx 0.46$ with a central momentum $k_{0}=\pi / 2-0.5 \sim k_{F}-7 \sigma_{0}$ and a width of $\sigma_{0} \sim 0.053$. The calculations were carried out with $N_{\text {cut }}=2500$ states kept per DMRG block in the full and adaptive time dependent DMRG. One clearly

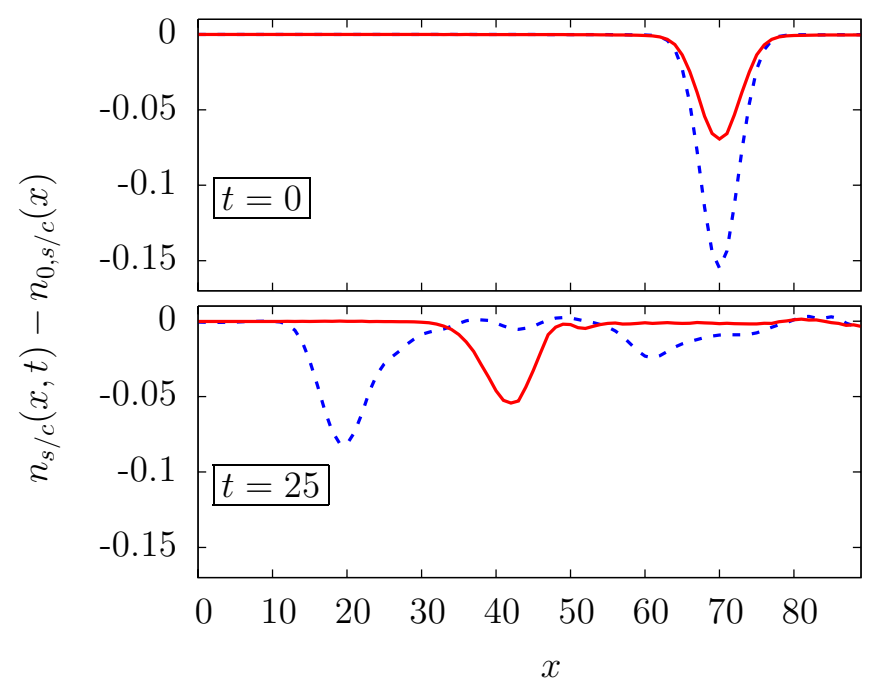

FIG. 3: (Colour online) Spin and charge density (see legend of fig. 2) in a Hubbard model at time $t=0$ (upper diagram) and $t=25$ (lower diagram) show spin-charge separation. This is data from a td-DMRG simulation on 90 sites system with $N_{\uparrow}=41-1, N_{\downarrow}=41$ electrons and a Hubbard interaction of $U=4$.

observes SCS driven by the Hubbard interaction between $t=0$ (upper diagram) and $t=25$ (lower diagram). We extract the group velocities from the main peaks to be $v_{S}=1.12 \pm 0.05$ and $v_{C}=2.03 \pm 0.05$. The velocity is constant through out the simulation and the error stems from the read-off error. The one dimensional Hubbard model can be solved exactly [23]. From the analytic expression by Coll [24], the spin and charge velocity of the low energy spinons and holons can be derived as done, e.g. by Schulz [25]. The excitation's momentum of $k_{0}$ corresponds to a band filling of $n=0.68$ in units of the hopping. Given the error estimate above, it is accurate enough to read off a charge velocity of $v_{C \text {, one holon }} \sim 2.05$ and $v_{\text {S.one spinon }} \sim 1.15$ (cf. figure 11 of the first paper of Ref. [25]). Although we are with 90 sites far from simulating the thermodynamic limit, the group velocities of our spin and charge density fit remarkably well to the Bethe Ansatz results for the velocity of one spinon and one holon. A similar good agreement was found for a spinless Luttinger liquid simulation [18].

Our electronic excitation with a definite momentum $k_{0}$ created a unidirectional running peak in the noninteracting system. Here, however, our initial excitation creates both a right and a left moving density peak in the charge sector. At time $t=25$, the right moving charge peak has already been reflected on the right wall and is located at around $x=60$. The spin density, in contrast, displays predominantely a left moving peak. Finally, there is charge density accumulation trailing the main charge peak and at the location of the spin peak. 
These might be more complicated features of the interaction or result from the finiteness of the system as in the non-interacting example.

Following the previous line of arguments, it seems reasonable to do a transport setup that uses non-interacting leads as a starting point. We let this excitation travel through an interacting region where SCS occurs and then let the resulting excitations travel again into a noninteracting area. In the starting lead our electronic excitation is well understood. When this excitation travels into the interacting region, we know the momentum and energy of the injected wave packet (one electron or one hole) but the questions arise: what kind of excitation leaves the structure and what can be measured on the opposite (non-interacting) lead?

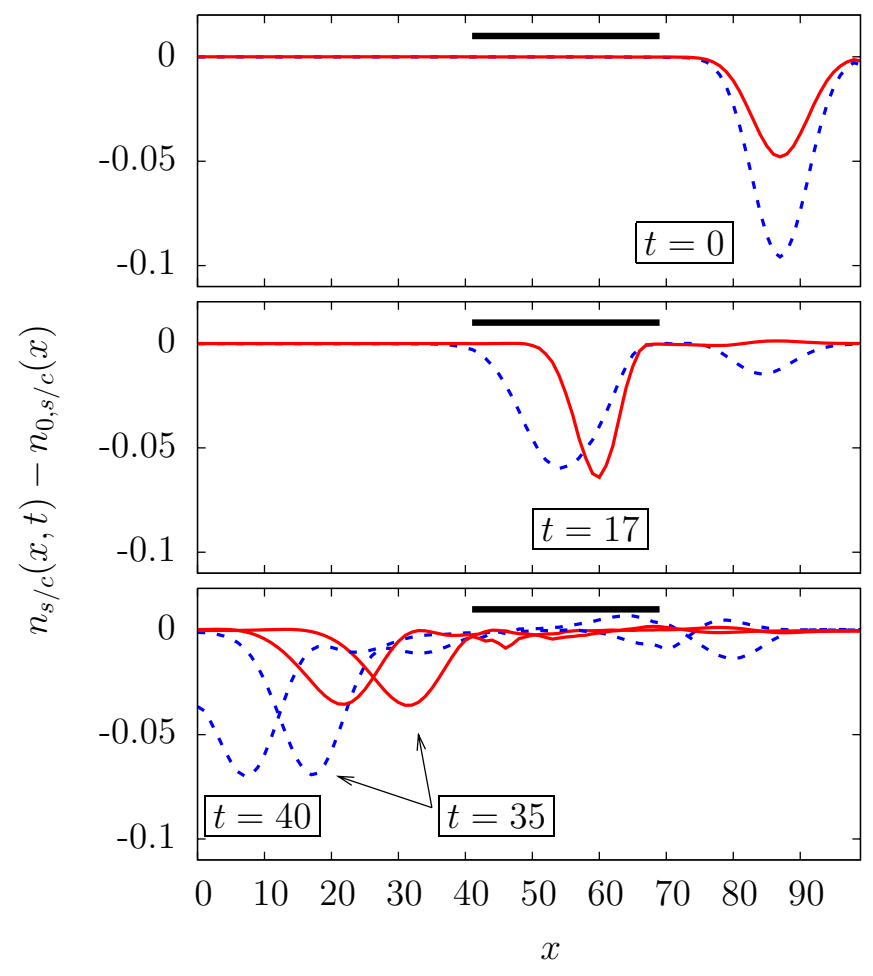

FIG. 4: (Colour online) Excitation in a transport experiment: a created hole in the right lead (upper diagram, at $t=0$ ) passes the interacting nanostructure (black bar) undergoing SCS (central diagram, at $t=17$ ). At $t=35$, 40 (lower diagram) spin and charge densities travel independently with equal velocity in the left lead.

We use a system of $M=100$ sites, split into 41,29 and 30 sites for the different areas and a total of $N_{\uparrow}=48-1$, $N_{\downarrow}=48$ electrons. The corresponding Hamiltonian reads

$$
\begin{aligned}
H_{\text {transport }}= & \sum_{\sigma=\uparrow, \downarrow} H_{\mathrm{tb}, \sigma}+V_{\text {gate }} \sum_{x=42 . .70}\left(n_{x, \uparrow}+n_{x, \downarrow}\right) \\
& +U \sum_{x=42 . .70}\left(n_{x, \uparrow}-\frac{1}{2}\right)\left(n_{x, \downarrow}-\frac{1}{2}\right),
\end{aligned}
$$

where $H_{\mathrm{tb}, \sigma}$ was defined in (11) and $U=2.5$. A gate voltage on the interacting area levels the Fermi surface to a half-integer number of up and down electrons on the structure $\left(N_{\uparrow}=N_{\downarrow}=12.5\right)$ and minimises reflections into and out of the area (see figure 44). This local chemical potential $V_{\text {gate }}$ in (7) was pre-calculated in a self-consistent way. Thus we have a Hubbard model at $\sim 0.43$-filling in the central (black bar) area and half-filled tight-binding leads. We also chose parameters $k_{0}=0.43 \pi-2 \sigma_{0}, \sigma_{0}=0.03$ to maximise tunnelling while staying as close as possible to a quasi-linear dispersion. The number of states kept was $N_{\text {cut }}=2500$. Figure 4 shows the time evolution of spin and charge densities for several times. There are reflections of charge density at both edges of the structure. They are suppressed by the choice of the chemical potential as discussed above. The majority of spin and charge density transmits through the nanostructure. SCS clearly happens inside the interacting region (central diagram at $t=17$ ) and SCS remains intact after leaving the interacting area into the opposite lead (lower diagram at $t=35$ and $t=40$ ). Here spin and charge now travel along separately but again with identical constant velocity.

\section{Conclusions}

In our simulation of a microscopic experiment on an interacting nanostructure we saw that the spin and charge excitation of an injected electron can be separated as it is known to happen in one-dimensional interacting systems. The spin and charge separation can be directly observed by a time-resolved measurement of the spinpolarised density. There is no need for spin and charge to recombine to a complete electron (hole) for a measurement of a single electron (hole). Note that the charge and spin excitations in the non-interacting leads are valid excitations of the Fermi liquid. They are not elementary excitations of the Fermi liquid, instead they are a composition of (at least) two excitations of the Fermi liquid. As an example, think of the combinations $\left(c_{k, \uparrow}^{\dagger} \pm c_{k, \downarrow}^{\dagger}\right)$. However the resulting distribution of separated densities originated from a single particle injection into the Fermi sea. Thereby, we confirm for the Hubbard model, what Safi and Schulz [10] sketched for a low-energy Luttinger liquid transport setup with restricted interaction. It is interesting that our small system already shows the fingerprint of Luttinger Liquid description expected in the thermodynamic limit. Also note that even for highly energetic incoming electrons we find the spin-charge separation, albeit obscured by band effects. With the tdDMRG method even more realistic models, like the extended Hubbard model with a finite next-nearest neighbour interaction could be investigated.

Experimentally, the measurement of the densities would have to happen on a time scale which is set by the Fermi velocity and the length of the interacting system. For nanoscaled systems and metallic excitation velocities tens of Terahertz resolution would be required, which might be achieved using optical techniques. Low- 
ering the Fermi, holon and spinon velocities would enhance the detection possibilities. It is of general interest to know, whether a series of quantum dots or any other strongly correlated system with a much smaller Fermi velocity can provide the ingredients for the realisation of such a transport setup.

\section{Acknowledgments}

We thank Dmitry Aristov, Rafael Molina, Ronny Thomale, and Peter Wölfle for valuable discussions and we acknowledge the support by the Center for Functional Nanostructures (CFN), project B2.10. The DMRG calculations have been performed on HP XC4000 at Steinbuch Center for Computing (SCC) Karlsruhe under project RT-DMRG.
[1] J. Voit, Rep. Prog. Phys. 58, 977 (1995).

[2] B. I. Halperin, J. Appl. Phys. 101, 081601 (2007).

[3] O. M. Auslaender et al., Science 308, 88 (2005).

[4] N. Tombros, S. J. van der Molen, and B. J. van Wees, Phys. Rev. B 73, 233403 (2006).

[5] M. Bockrath et al., Nature (London) 397, 598 (1999).

[6] B. J. Kim et al., Nature Phys. 2, 397 (2006).

[7] R. Claessen et al., Phys. Rev. Lett. 88, 096402 (2002).

[8] M. Sing et al., Phys. Rev. B 68, 125111 (2003).

[9] H. Benthien, F. Gebhard, and E. Jeckelmann, Phys. Rev. Lett. 92, 256401 (2004).

[10] I. Safi and H. J. Schulz, Phys. Rev. B 59, 3040 (1999).

[11] E. A. Jagla, K. Hallberg, and C. A. Balseiro, Phys. Rev. B 47, 5849 (1993).

[12] M. G. Zacher, E. Arrigoni, W. Hanke, and J. R. Schrieffer, Phys. Rev. B 57, 6370 (1998).

[13] C. Kollath, U. Schollwöck, and W. Zwerger, Phys. Rev. Lett. 95, 176401 (2005).

[14] P. Schmitteckert, in High Performance Computing in Science and Engineering '0\%, edited by W. E. Nagel, D. B. Kröner, and M. Resch (Springer, Berlin, 2007), pp. 99106.

[15] P. Schmitteckert and G. Schneider, in High Performance Computing in Science and Engineering '06, edited by W. E. Nagel, W. Jäger, and M. Resch (Springer, Berlin,
2006), pp. 113-126.

[16] E. Boulat, H. Saleur, and P. Schmitteckert, Phys. Rev. Lett. 101, 140601 (2008).

[17] S. R. White, Phys. Rev. Lett. 69, 2863 (1992).

[18] P. Schmitteckert, Phys. Rev. B 70, 121302 (2004).

[19] G. Vidal, Phys. Rev. Lett. 93, 040502+ (2004).

[20] S. R. White and A. E. Feiguin, Phys. Rev. Lett. 93, 076401 (2004).

[21] A. J. Daley, C. Kollath, U. Schollwöck, and G. Vidal, J. Stat. Mech. 2004, P04005 (2004).

[22] R. M. Noack and S. R. Manmana, AIP proceedings, edited by A. Avella and F. Mancini (AIP, ADDRESS, 2005), Vol. 789, pp. 93-163;

R. M. Noack and S. R. Manmana, Diagonalizationand Numerical Renormalization-Group-Based Methods for Interacting Quantum Systems (2005), http://arxiv.org/abs/cond-mat/0510321

K. A. Hallberg, Advances in Physics 55, 477 (2006);

U. Schollwöck, Rev. Mod. Phys. 77, 259 (2005).

[23] E. H. Lieb and F. Y. Wu, Phys. Rev. Lett. 20, 1445 (1968).

[24] C. F. Coll, Phys. Rev. B 9, 2150 (1974).

[25] H. J. Schulz, Int. J. Mod. Phys. B 5, 57 (1991); http://arxiv.org/abs/cond-mat/9302006 